\title{
LO STATUTO DEL COLLEGIO DEI DOCTORES ARTIUM ET MEDICINAE DELL'UNIVERSITA' DI PAVIA DEL 1409: LA COPIA COMPLETA RITROVATA ${ }^{\circ}$
}

\author{
Nota del m.e. DARIO MANTOVANI (*)
}

(Adunanza del 17 gennaio 2013)

SunTO. - In questa nota è descritta la copia dello statuto del Collegio dei dottori in Arti e Medicina dello Studium pavese (promulgato nel 1409) recentemente individuata presso il Museo per la Storia dell'Università di Pavia. Il manoscritto, il cui nucleo più antico risale al XVI secolo, testimonia il testo completo dello statuto, a differenza della copia settecentesca precedentemente nota, in cui erano omesse dieci delle ventisei rubriche. Il manoscritto contiene inoltre le modifiche successive e gran parte dei nomi dei membri del Collegio dal 1409 al 1762. Particolare attenzione è qui rivolta all'impianto istituzionale presupposto dallo statuto, a commento della trascrizione di due brani di particolare rilevanza storica, ossia la formula di approvazione e il capitolo che determina i libri su cui era basato l'esame di laurea.

$* * *$

ABSTRACT. - This paper describes the copy of the Statuta Collegii doctorum Artium et Medicinae issued for the University of Pavia in 1409, recently rediscovered at the Pavia University History Museum. The most ancient part of the manuscript, which dates to $16^{\text {th }}$ century, witnesses the complete text of the $15^{\text {th }}$ century Statuta, whereas the copy

- La nota presentata all'Istituto il $17 / 1 / 2013$ dava la prima notizia del ritrovamento; la si pubblica qui, integrandola con alcuni approfondimenti tratti da un successivo studio (Il Collegio dei dottori in Arti e Medicina di Pavia in Età spagnola. Notizie dal manoscritto ritrovato, in Mantovani, D., a c. di, Almum Studium Papiense. Storia dell'Università di Pavia, 1.II, Milano, Cisalpino, 2013, 895-910).

(*) Università degli Studi di Pavia, Dipartimento di Giurisprudenza, Pavia, Italy. E-mail: dario.mantovani@unipv.it 
previously known, which dates to $18^{\text {th }}$ century, lacks of ten chapters. The manuscript also contains subsequent amendments and most of the names of the members of the Collegium from 1409 to 1762. Particular attention is paid, in this presentation, to the institutional framework implied by the Statuta; with that aim, its formula of approval (dated February 1409) and the chapter which lists the books on which doctoral examinations in Pavia were based are transcribed.

\section{IL MANOSCRITTO}

Nel luglio 2012, in un volume pergamenaceo conservato presso il Museo per la Storia dell'Università di Pavia, ho potuto riconoscere il testo completo dello statuto del Collegio dei dottori in Arti e Medicina promulgato nel 1409. Il manoscritto riporta anche le principali modifiche e disposizioni successive, dal 1433 fino al 1738; inoltre, ci fa conoscere i nomi dei doctores collegiati in un arco di tempo che s'estende, sia pure a intermittenza, dal 1409 al 1762.

Il volume pergamenaceo risulta presente già nell'inventario del 10 marzo 1951 del Museo (n. 2113), correttamente etichettato come copia dello statuto. ${ }^{1}$ Quel che non era stato visto è che contiene un testo diverso da quello edito da Rodolfo Maiocchi nel 1915 e da Luigi Franchi nel 1925. ${ }^{2}$ I due editori novecenteschi - gli ultimi a essersi occupati dello statuto del Collegio di Arti e Medicina - avevano infatti utilizzato un testimone più recente, del XVIII secolo, oggi conservato presso la Biblioteca Universitaria di Pavia. ${ }^{3}$ La copia settecentesca fu eseguita

1 Statuta, Ordines et Nomina Collegii doctorum Artium et Medicinae felicis Studii Almae Urbis Papiensis (1409-1762); d'ora in poi citato come MSUPv, Statuta, Ordines et Nomina Collegii doctorum Artium et Medicinae Studii Papiensis.

2 Rispettivamente Codice diplomatico dell'Università di Pavia, II.1, Pavia, Fusi, 1915, 111-118 (doc. 183); Statuti e Ordinamenti della Università di Pavia dall'anno 1361 all'anno 1859, Pavia, Tipografia Cooperativa, 1925, 119-143. Una fotografia, anteriore all'identificazione, compariva nel catalogo della mostra Pavia e le svolte della scienza, a c. di Mazzarello, P. - Fregonese, L., Pavia, CLU, 2011, 8.

3 Statuta et Ordines Collegii nob. DD. Physicorum Papiae, S.R.I. Comitum et Equitum 1409-1791: BUPv, Ticinesi, 748 (il titolo risulta nel catalogo, non nel ms.); di 71 pp., contiene alle pp. 1-26 lo statuto del 1409 (segni a matita blu sono apposti in corrispondenza delle rubriche omesse, traccia di una posteriore ricollazione con l'esemplare completo; vd. Figg. 1-2). Seguono di stessa mano estratti degli aggiornamenti normativi fino all'8 gennaio 1619. A p. 72 inizia un decreto di Maria Teresa del 7 maggio 1751, con il quale (oltre a confermare il privilegio del $1667 \mathrm{di}$ Leopoldo I, che insigniva del titolo di 
probabilmente in occasione di una disputa che aveva coinvolto il Collegio dei medici di Pavia, in seguito a un decreto di Maria Teresa d'Austria, del 7 maggio 1751, con cui si regolavano i criteri d'accesso al Collegio. Questo genere di controversie s'inquadrava nel contenzioso aperto fra il governo austriaco, che tentava una liberalizzazione della società, e le corporazioni che resistevano abbarbicate alle posizioni di rendita. Chi copiò dopo la metà del Settecento il volume antico - verosimilmente per allegarlo a sostegno delle proprie rivendicazioni - lo abbreviò, limitandosi a trascrivere le disposizioni che ancora potevano essere attuali e rilevanti nella contesa. Le edizioni moderne, che si appoggiano a quel testimone settecentesco, sono pertanto incomplete: il manoscritto conservato al Museo rivela che lo statuto del 1409 era formato da ventisei rubriche, dieci in più di quelle finora note (ed elimina così i sospetti ricorrenti che la redazione del 1409 fosse in qualche modo ab origine imperfetta; come vedremo, fu anzi adottata come modello da altre Università). ${ }^{4}$ Del tutto inedita è poi la lista dei componenti del Collegio, annotata annualmente dal priore che lo presiedeva, da Martino de' Medici nel 1409 a Giuseppe Carena nel 1762. ${ }^{5}$

Equites aurati et comites palatini i dottori fisici di Pavia) si statuiva che in futuro non si sarebbe potuto cooptare nel Collegio se non il laureato a Pavia che «ex antiqua et antiquitus nobili familia ipsius civitatis, vel loci eiusdem Principatus originem duxerit», intendendosi famiglia che avesse abitato per cento anni a Pavia o nel principato «decorose et nobiliter» (cioè se il padre o nonno non avesse esercitato mestiere vile). Segue di altra mano una petizione del 1783, il cui redattore leggeva proprio il volume oggi conservato al Museo, perché menziona l'ordine di Filippo II del 1554 indicando che si trova al «fol. 26 tergo», che corrisponde alla paginazione del volume in questione. Il ms. settecentesco (BUPv, Ticinesi, 748) termina con un carteggio sul divieto di indossare la toga da parte dei collegiati: una lettera che menziona Alessandro Volta è mutila.

4 Esiste un'altra trascrizione completa dello statuto del 1409, già appartenuta a Carlo Magenta e ora conservata in BUPv, Ticinesi, 413 (pp. 1-19), anch'essa evidentemente sconosciuta a Maiocchi e Franchi e ai successivi studiosi; la copia (priva degli aggiornamenti posteriori al 1409 e degli elenchi dei doctores) omette anche la subscriptio finale.

5 Il manoscritto è composito (vd. per i dettagli infra) e anche le liste riflettono questa varietà: nella prima parte del volume, alla lista dell'anno 1409 (c. $8 r-v$ ) seguono a distanza quelle del 1453 (c. 19r-v) e del 1487 (c. 24r-v), frammiste a liste del turno di rotazione alla carica di priore (circulus), fino al 1522 (c. $25 r$-v). Nella seconda parte, si riprende con la lista del 1585 (c. 40r) e del 1593 (c. 40v); in quella che si può definire terza parte gli elenchi annuali si infittiscono progressivamente, e sono pressoché costanti dalla fine del Seicento al 1762. 
Il volume, rilegato, consta di 72 fogli. ${ }^{6} \grave{E}$ difficile stabilire in che modo sia stato formato. Possiamo confrontarlo con il parallelo volume che contiene lo statuto dei dottori giuristi dello Studium, anch'esso conservato presso il Museo, ma che, a differenza di quello dei medici, era noto e edito a stampa fin dal $1735 .{ }^{7}$ Lo statuto dei giuristi fu promulgato nel 1395; il manoscritto disponibile è un exemplum, cioè una copia autentica dell'originale, eseguita (rubriche in inchiostro rosso comprese) dal bidello Pantaleone da Crema nel 1400, su incarico del priore del Collegio di quell'anno, Antonio Guastoni. ${ }^{8}$ Seguono alcune aggiunte normative, e l'elenco dei membri del Collegio del 1400. Da quel punto in poi nel volume si srotola la matricola dei doctores collegiati, stilata a partire dal 1410 sotto il priorato di Didimo Cani, in ordine cronologico di ammissione; ' la matricola subisce un'interruzione nel 1506, riprende nel 1526 (dopo la battaglia di Pavia) e prosegue fino al $1680 .{ }^{10}$ Si tratta, insomma, del libro

6 Il primo foglio (carta di guardia, il cui recto è utilizzato per annotare l'elenco del 1750) e il secondo (contenente l'indice dei successivi 8 fogli) e gli ultimi 10 non sono numerati. L'ultima carta numerata, la $60^{\mathrm{a}}$, reca l'elenco dei membri del Collegio nel 1731 (recto) e 1732 (verso). Nel seguito, per comodità, mantengo la numerazione del ms., designando le prime due carte non numerate come [a] e [b].

7 MSUPv, Statuta, Ordines et Nomina Collegii doctorum utriusque iuris Studii Papiensis. La prima edizione è in Statuta et Ordines tam veteres, quam novi Illustrissimi, E Venerandi Collegii Illustrissimorum Dominorum Doctorum Nobilium, E Judicum inclitae Civitatis Papiae, Papiae, Ex Typographiae Joannis Benedicti Rovedini sub signo Jesu, 1735; la più recente risale a Statuti e Ordinamenti, Pavia, Tipografia Cooperativa, 1925, 93-118. Entrambe non contengono la lista dei doctores.

8 «Ego Pantaleonus de Crema filius quondam Zambonini de Plaranicis de Crema, Bidellus generalis almi Studii Papiensis ac specialis bidellus reverendissimi colegii [sic] dominorum doctorum utriusque Iuris inclite civitatis Papie, praedicta statuta scripsi ac rubricavi. Anno 1400 de mense madii de mandato et impositione spectabilis egregii legum doctoris domini Antonii de Guastonibus tunc prioris collegii prelibati» (MSUPv, Statuta, Ordines et Nomina Collegii doctorum utriusque Iuris Studii Papiensis, c. $13 v=$ Statuti e ordinamenti, 111 - con qualche variante). È curioso che la copia sia stata eseguita in un anno in cui lo Studium era temporaneamente trasferito a Piacenza.

9 Il Collegio del 1410 è ivi, c. 18v: colpisce che la lista si apra con il nome di Baldo degli Ubaldi, morto il 28 aprile 1400. L'elenco cronologico delle ammissioni (c. 19r-v) risale retrospettivamente al 1391 (fino al 1410); riprende poi dal 1413 (c. 20r).

10 L'elenco del 1400 si trova ivi, c. $17 r$ (segnalo en passant che la numerazione antica dei fogli del ms. è irregolare: in corrispondenza dell'anno 1652, dopo la c. 94 la foliazione prosegue con la c. 75 ; in corrispondenza del 1664, dopo la c. 83 prosegue con la c. 64). Dopo la c. 81 (bis), un fascicolo distinto contiene gli elenchi del 1591 e 1592 e alcune disposizioni. 
ufficiale del Collegio giurista, redatto nel 1400 (cioè solo cinque anni dopo la promulgazione dello statuto) e tenuto aggiornato pressoché senza soluzione di continuità fino quasi al termine dell'età spagnola. ${ }^{11}$

Il volume del Collegio dei doctores in Arti e Medicina, pur analogo nei suoi contenuti a quello dei giuristi, se ne distingue tuttavia sotto vari aspetti. Non reca alcuna indicazione circa la data della sua confezione. Inoltre, la registrazione dei doctores non ha la natura di una matricola continua che indichi la data d'ingresso nel Collegio, come avviene nel caso dei giuristi: si tratta invece (pur con qualche eccezione) degli elenchi di tutti i membri del Collegio in un dato anno. ${ }^{12}$ Infine, mentre il volume del Collegio dei giuristi procede in modo cronologicamente continuo, quello dei dottori artisti e medici si presenta molto disordinato nella parte relativa agli elenchi annuali, che spesso appaiono disposti alla rinfusa (o secondo una logica che sfugge). Capita così, ad esempio, che il primo elenco di doctores registrato dopo la parte propriamente statutaria riguardi l'anno 1682, e preceda l'elenco del 1632 (risp. cc. $30 v$ e $31 v$ ). In vari casi quest'accavallamento si può spiegare in modo semplice: chi voleva annotare nel volume l'elenco relativo al suo anno cercava una pagina in cui fosse rimasto uno spazio bianco (come è il caso appunto per l'elenco del 1682). Altre volte è meno facile capire per quale ragione l'elenco più recente preceda, in una stessa pagina, quello più antico, come avviene ad esempio nella c. $32 v$ per il 1672 e il $1635 .{ }^{13}$

11 Di questo volume (o almeno della sua prima parte) è menzione nella Relazione inviata il 21 agosto 1768 dal Collegio dei Nobili Giureconsulti Giudici, ed Avvocati, e Conti Palatini di Pavia al Governo austriaco (Pavia - Archivio di Stato, Università, Collegio dei Giureconsulti, cart. 47), dove, dopo avere lungamente descritto la perdita dei documenti causata dal sacco del 1527 (e il trasporto degli archivi a Digione), si afferma che «consta per somma ventura un volumetto in pergamena su cui sonovi li Statuti del collegio delli Dottori e Studio Generale, il quale scampò, non si sa come, dalla investigazione de persecutori, e nel quale sonovi segnati giorno, mese, ed anno dell'ingresso di alcuni Dottori de primi tempi del rinnovato Studio generale (Matricola delli Dottori del Colleggio compilata nel tempo del priorato d'Aula del Sig.r Didimo de Cani, nell'anno 1410)».

12 Vd. supra.

$13 \mathrm{Vd}$. anche, ad. es., la c. $37 r$, che reca gli elenchi del 1677 e del 1678, seguiti da annotazioni relative ai priorati degli anni 1649, 1657, 1662. In casi del genere, non si può escludere che la lista più antica (collocata dopo un'altra più recente nella stessa facciata) sia stata ricopiata più tardi. Ciò implicherebbe che il volume non sia stato aggiornato anno per anno e sia stato formato in modo meno lineare. Va contemplata anche la possibilità che il volume sia frutto della rilegatura di fogli in origine sparsi. 
Stabilire la datazione del nostro manoscritto è dunque arduo. Di sicuro, è il prodotto stratificato di alcuni secoli, fino al 1762 quando si arresta la registrazione annua dei doctores in Arti e Medicina.

Considerando la struttura qui rapidamente descritta, la datazione dello strato più antico è da collocare a metà circa del XVI secolo (al più tardi alla fine del secolo).

Lo statuto del 1409, le successive norme quattrocentesche e gli elenchi dei doctores fino al 1522 (cc. $1 r$-25r) sono, infatti, vergati da una stessa mano. La scrittura è un'italica molto elegante, del tipo talvolta definito italica testeggiata. ${ }^{14}$ Anche l'indice premesso al volume (c. [b]) si riferisce (solo) al contenuto di questi primi 25 fogli, confermandone in un certo senso l'unitarietà. ${ }^{15}$ Quest'insieme non può dunque essere anteriore al 1522. A questa prima parte fanno seguito alcune pagine che si riferiscono a documenti datati fra il 1550 e il 1593 (cc. 25r-30v), di una mano di poco posteriore e comunque simile alla prima. Le iniziali ornate che occorrono sono di due tipi: 1) filigranate, retaggio della tradizione del libro bassomedievale e molto in uso in epoca umanistica (della prima mano); 2) riquadrate e decorate (della seconda mano), più tipiche del libro umanistico "nuovo" in antiqua (la minuscola carolina rielaborata dagli umanisti). ${ }^{16}$

Chiudono il volume pagine di varia grafia e data.

Parrebbe dunque che la prima parte del manoscritto - di cui è responsabile la mano più antica - sia da datare fra il 1522 e il 1550, mentre il seguito si sia formato nel tempo, dal 1550 al $1762 .{ }^{17}$ Considerato che proprio nell'intervallo fra il 1522 e il 1550 cade il terribile sacco inflitto alla città nel 1527 dalle truppe francesi comandate da Odet de Foix, visconte di Lautrec, che causarono brutale dispersio-

${ }^{14}$ Vd. Cherubini, P. - Pratesi, A., Paleografia latina. L'avventura grafica del mondo occidentale, Città del Vaticano, Scuola Vaticana di Paleografia, Diplomatica e Archivistica, 2010, 627.

15 Per l'esattezza, la prima scrittura della c. [b] indicizza le cc. $1 r-24 r$, ossia lo statuto e gli aggiornamenti successivi, fino a una lettera ducale del 1478. Anche le cc. $24 r-25 v$, che contengono gli elenchi dei doctores fino al 1522, sembrano scritte dalla stessa mano. grafica.

16 Ringrazio i colleghi Paolo Rosso e Serena Ammirati per la consulenza paleo-

17 Si tratta solo di un'ipotesi di massima. Le variabili sono molte: ad esempio, la seconda parte (che comincia con una disposizione del 1550) potrebbe essere stata scritta intorno al 1593, anno cui risalgono vari atti copiati alle cc. 28r-29r (il che può fare oscillare la composizione della prima parte fra il 1522 e il 1593). 
ne e danneggiamento anche degli archivi, può essere suggestivo ritenere che la copia dello statuto dei dottori in Arti e Medicina (vergato dalla prima mano) sia stata eseguita dopo quell'anno e prima del 1550, quando finalmente l'Università riprendeva il suo corso. ${ }^{18}$

\section{La data dello Statuto E Il Collegio come commissione DI LAUREA}

L'edizione del manoscritto offrirà solida base documentaria alla storia normativa e all'indagine prosopografica. ${ }^{19}$ In questa sede, ci limitiamo a trascrivere due passaggi di particolare rilievo storico, ossia la formula di approvazione dello statuto e il capitolo XII che elenca i libri oggetto di studio ed esame all'Università di Pavia nel XV secolo. La trascrizione darà l'occasione anche di riepilogare le funzioni del Collegio dei dottori.

La formula di approvazione fa conoscere il mese (febbraio) di quel 1409 in cui lo statuto fu riformato: periodo singolarmente tormentato per il ducato di cui era signore Giovanni Maria Visconti, dilaniato da una guerra fra bande, mentre il giovanissimo Filippo Maria risiedeva a Pavia, che il padre gli aveva assegnato come contea. Soprattutto, la formula restituisce i nomi dei dottori che parteciparono all'approvazione. Data e nomi erano omessi dalla copia utilizzata da Maiocchi. Questa la trascrizione:

Anno a nativitate domini $\mathrm{MCCCC}^{\circ}$ nono de mense Februarij / fuerunt reformata antescripta Statuta Collegij Artium / et Medicine doctorum; per Venerabiles et Egregios Viros Artium / et Medicine doctores, videlicet dominum Magistrum Martinum de Medi/cis de Viqueria dicti Collegij tunc priorem et dominum Magistrum An/tonium de Vachis de Bremide. Ac confirmata per omnes docto/res utriusque Collegij quorum nomina inferius describuntur: / Nomina doctorum utriusque doctorum Collegij sunt ista videlicet / Magister Martinus de Medicis suprascriptus artium et medicine doctor / Magister

$18 \mathrm{Se}$, invece, la prima parte dovesse essere datata alla seconda metà del Cinquecento, secondo quanto osservato nella nt. prec., l'iniziativa di copiare lo statuto e gli ordini potrebbe essere ricollegata alla riforma del 1554, che trasformò il Collegio dello Studio in Collegio nobile (su questa metamorfosi, vd. ampiamente nel seguito).

19 Rinvio per una valutazione al saggio citato supra nt. ' ; l'edizione del testo sarà pubblicata dal Centro per la Storia dell'Università di Pavia, a cura di chi scrive. 
Antonius de Vachis artium et medicine doctor / Magister Francischinus de Strazapatis artium et medicine doctor / Magister Matheus de Vitudono artium et medicine doctor / Magister Bethinus de Biscossis artium et medicine doctor [c. 8r] / Magister Johannes Francischus de Balbis artium et medicine doctor / Magister Jacobinus de Panizarijs artium et medicine doctor / Magister Christophorus de Brixia artium et medicine doctor / Magister Gaspaxinus de la Mayrola artium et medicine doctor / Magister Antonius de Marzarijs de Castronovo artium et medicine doctor / Magister Johannes de Marliano ordinis heremitani Sancti Augustini artium et medicine doctor / Magister Sanctinus de Folpertis artium et [sic] doctor / Magister Aricus de Colombis de Sallis artium et medicine doctor / Magister Apollinarius de Cremona artium et medicine doctor / Magister Thibaldus de Madijs artium et medicine doctor / Magister Petrus de Montralcino artium et medicine doctor / Magister Syrus de Rubeis artium et medicine doctor / Magister Marchus de Nebiola artium doctor / Magister Marchus de Lumenachis de Papia medicine doctor / Magister Antonius de Treviso artium et medicine doctor / Magister Nicolaus de Cremona artium et medicine doctor / Magister Johannes Martinus de Carisio artium et medicine doctor / Magister Jacobus de Ferrarijs de Gambolato artium et medicine doctor / Magister Johannes Nicolla de Buris Papiensis artium et medicine doctor [c. $8 v$ ].

L'elenco contiene evidentemente i componenti del Collegio dei dottori in Arti e Medicina al momento in cui si davano un nuovo regolamento.$^{20}$ Conviene ricordare che lo Studium, in età medievale $\mathrm{e}$ fino all'età austriaca, era costituito da due Facultates, cioè da due campi di sapere, il Diritto da una parte e le Arti e la Medicina dall'altro. Nell'ambito di ciascuna Facultas operavano due fondamentali organismi corporativi, il Collegium dei doctores e l'Universitas degli studenti. ${ }^{21}$ Distinto dall'uno e dall'altra era il corpo dei docenti, di nomina pubblica annuale (in età spagnola, di competenza del Senato di Milano). Dunque a Pavia, oltre ai professori veri e propri, operavano il Collegium doctorum utriusque Iuris Studii Papiensis, con la

20 Si noti che nell'elenco si indica precisamente se il dottorato di ciascuno è in Arti e in Medicina oppure in una soltanto delle materie, e nell'intestazione si parla di utrumque Collegium, a significare una sorta di irrisolta duplicità fra i due insiemi.

${ }^{21}$ Il terzo settore disciplinare, la Teologia, ha strutture peculiari, in parte esterne allo Studium generale: vd. Negruzzo, S., La Facultas Theologiae, in Mantovani, D., a c. di, Almum Studium Papiense. Storia dell'Università di Pavia, 1.I, Milano, Cisalpino, 2012, 609-630 e Bernuzzi, M., L'insegnamento della Teologia. Discipline e strumenti, ivi, 1.II, 1151-1186. 
relativa Universitas scolarium iuristarum e il Collegium doctorum Artium et Medicinae Studii Papiensis, con la relativa Universitas scolarium artistarum et medicorum. Ciascuno di questi organismi, proprio in ragione del carattere corporativo, doveva darsi delle regole, appunto uno statuto (nome collettivo che designa l'insieme delle singole norme, gli statuta). Lo statuto disciplinava i requisiti di ammissione, istituiva le cariche sociali (il rettore di ciascuna Universitas era ovviamente uno studente; i Collegia si davano invece un priore, che era a turno uno dei doctores), determinava i poteri e attribuiva i compiti. Il manoscritto di cui ci occupiamo restituisce, come s'è più volte detto, lo statuto del Collegium doctorum Artium et Medicinae felicis Studii urbis Papiensis: i requisiti di ammissione e i compiti di tale Collegio possono istruirci sull'evoluzione sociale e organizzativa della Facoltà medica.

Primo requisito di ammissione al Collegio era il dottorato in Medicina o in Arti o in entrambe le Facoltà. ${ }^{22}$

Non avrebbe potuto essere altrimenti, visto che la competenza essenziale dei dottori in Arti e Medicina (come del parallelo Collegio dei giuristi) era di fungere da "commissione di laurea". I doctores prendevano parte all'esame privato di rigorosa verifica delle competenze dei laureandi e alla successiva cerimonia pubblica (conventus) presieduta dal vescovo, che culminava nel conferimento delle insegne di dottorato.

Il ruolo di commissione di laurea si riverberava anche sulla struttura del Collegio. Fin dal 1409, fu sancito che il Collegio fosse costituito da un nucleo di quattordici membri effettivi (doctores numerarii) e da una cerchia indefinita di membri ulteriori, supernumerarii. Solo i numerarii erano a tutti gli effetti i commissari di laurea. I collegiati supernumerarii potevano anch'essi assistere alle sedute, ma

22 Statutum sextum: MSUPv, Statuta, Ordines et Nomina Collegii doctorum Artium et Medicinae Studii Papiensis, c. $2 v$ (manca nel ms. del XVIII sec. e quindi nell'edizione di Statuti e Ordinamenti). Il neocollegiato (che doveva avere almeno vent'anni) doveva versare una somma non puramente simbolica, ma nemmeno esosa, ossia cinque fiorini, la moneta d'oro da 3,54 grammi che rappresentava la principale divisa di scambio in Europa. Per fare un confronto, nello stesso periodo in cui fu fissata la tariffa d'ingresso un docente di prestigio quale Biagio Pelacani percepiva come salario annuo duecento fiorini e il salario dei bidelli generali ammontava a cinquanta (Codice diplomatico, II.1, 84: doc. 139). 
non avevano diritto di esprimere il voto sul candidato né di spartirsi le propine che questi doveva alla commissione esaminatrice. ${ }^{23} \mathrm{Si}$ tratta dunque di un vero e proprio numero chiuso, finalizzato in primo luogo a garantire la serietà dell'esame e a lucrare i relativi ricavi. Le cifre in gioco, per la verità, non erano trascendentali, tuttavia potevano costituire un'entrata non disprezzabile (e, ad ogni modo, proprio perché non elevate, si cercava di non sminuzzarle ulteriormente). Il manoscritto consente di conoscere gli importi percepiti dai numerarii: sette fiorini per l'esame rigoroso (la verifica vera e propria della preparazione, certificata con la licentia) e sette per la cerimonia pubblica (il conventus) in cui il licentiatius riceveva finalmente il titolo e le insegne dottorali. ${ }^{24} \mathrm{~A}$ conti fatti, per la partecipazione a ciascuno di questi due atti accademici (necessari entrambi per arrivare al grado di dottore) ognuno dei quattordici doctores numerarii del Collegio percepiva mezzo fiorino. Immaginando che in un anno un doctor prendesse parte in media a quindici licentiae e ad altrettanti conventus, avrebbe percepito in totale quindici fiorini (sempre rapportandosi alle cifre del primo Quattrocento, e solo per avere un'idea di massima, la cifra poteva equivalere a una mensilità di un docente ben pagato). ${ }^{25}$

Ma non di sole propine è fatta una laurea. Fra le novità più importanti recate dal manoscritto del Museo è la rubrica dedicata alle materie su cui verteva l'esame (statutum duodecimum, c. 4r-v). Assente nella copia settecentesca e nelle edizioni moderne che da essa dipendono,

23 Statutum septimum: MSUPv, Statuta, Ordines et Nomina Collegii doctorum Artium et Medicinae Studii Papiensis, c. 3 r (=Statuti e Ordinamenti, 123). I supernumerarii non possedevano neppure l'elettorato passivo alla carica di priore del Collegio, che i numerarii ricoprivano a rotazione annua (circulus).

$24 \mathrm{Vd}$. rispettivamente statutum decimumsextum e vigesimum: MSUPV, Statuta, Ordines et Nomina Collegii doctorum Artium et Medicinae, cc. $5 v$ e $6 v$.

25 Ben più cospicua era la somma che spettava a ciascun promotore, otto fiorini (vd. ancora statutum vigesimum, ivi, c. 6v). Secondo lo statuto, i promotori (in caso di laurea in Medicina) dovevano essere il docente di Medicina teorica ordinaria mattutina e il docente di Pratica ordinaria le cui lezioni il candidato avesse seguito per maggiore tempo (rispetto, s'intende, agli eventuali docenti concorrenti della stessa materia). Il laureando poteva scegliere promotori aggiuntivi, purché membri del Collegio. In questo modo, i docenti potevano ottenere un'integrazione notevole rispetto al salario spettante per l'insegnamento (in alcuni anni si poteva arrivare alla metà); ciò spiega anche le rivalità fra docenti concorrenti, per accaparrarsi il maggior numero di studenti. 
permette di conoscere i contenuti didattici del percorso artistico e del percorso medico a Pavia, dal Medioevo all'età moderna: ${ }^{26}[\mathrm{vd}$. Fig. 3]

XII De assignatione punctorum. Item statuerunt quod scolari examinando in medicina assignentur puncta in libro Tegni Galieni et in libro Afforismorum unum quolibet [sic] per unum de doctoribus Collegij. In presentia domini Cancellarii vel eius Vicecancellarij aperiendo librum casu et punctando ante et retro duas cartas si forte aperitio caderet in loco ubi punctus $[$ sic] non esset ydoneus. In logica vero assignentur puncta in libro Posteriorum et in libro Periermenias si in sola logica debeat aliquis examinari. In naturali vero philosophia assignentur puncta in libro Phisicorum et in libro De anima, si in sola phisica debeat examinari; si vero in logica et in phylosophia simul examinatio fienda sit prout in maiori parte fit tunc assignentur puncta in libro Physicorum et in libro Posteriorum. In gramatica vero assignentur puncta in Prisciano maiori et in Modis significandi. In rethorica in libro Rethorice nove Tulij. In geometria, vero in libro Ellementorum Euclidis. In arithmetica in libro Arithmetice Boetii vel in Algorismo Johannis de Sacro boscho. In musica vero in libro Musice Boetij et in astronomia in tractatu de Spera vel in prima secunda tertia aut quarta dictione Almagesti Ptholomei.

26 «Assegnazione dei passi [da discutere all'esame]. Ancora stabilirono che allo studente da esaminare in Medicina siano assegnati i passi nel libro Tegni di Galeno

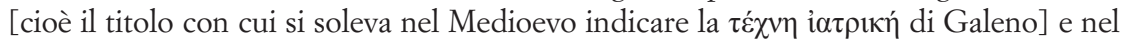
libro degli Aforismi di Ippocrate uno qualsiasi a opera di uno dei dottori del Collegio, in presenza del signor cancelliere o del suo vicecancelliere, aprendo il libro a caso, e puntando avanti e indietro due fogli nel caso l'apertura cada in un luogo ove non vi sia un punto idoneo. Nella Logica invece si assegnino passi nel libro degli Analitici secondi [di Aristotele, tradotti in latino] e nel libro dell'Interpretazione se il candidato debba essere esaminato solo in Logica. Nella Filosofia naturale invece siano assegnati punti nel libro dei Fisici e nel libro sull'Anima [di Aristotele] se debba essere esaminato solo in Fisica; se invece l'esame debba avvenire contestualmente in Logica e in Filosofia [naturale], come avviene nella maggior parte dei casi, allora si assegnino passi nel libro dei Fisici e nel libro degli Analitici secondi. In Grammatica invece si assegnino i punti nel Prisciano maggiore [Institutio de arte grammatica] e nei Modi dei significati [di Tommaso da Erfurt]. Nella Retorica, nel libro della Retorica nuova di Cicerone [Rhetorica ad Herennium]. In Geometria invece nel libro degli Elementi di Euclide. In Aritmetica nel libro di Aritmetica di Boezio o nell'Algorismo di Giovanni di Sacrobosco. In Musica invece nel libro di Musica di Boezio e in Astronomia nel trattato sulla Sfera [di Giovanni di Sacrobosco] o nella prima seconda terza o quarta sezione dell'Almagesto di Tolomeo». Nella trascrizione, ho normalizzato le maiuscole. 
Sotto forma di istruzioni rivolte ai commissari d'esame su come effettuare la scelta dei passi che il candidato doveva discutere, la rubrica contiene un vero e proprio piano di studi. Chi ambiva alla professione di medico di solito conseguiva quale primo titolo la laurea in una o più delle discipline inserite nel curriculum delle Arti: come lo stesso statuto osserva, la laurea più frequente comprendeva contemporaneamente la Logica e la Filosofia naturale («si vero in logica et in phylosophia simul examinatio fienda sit prout in maiori parte fit») ${ }^{27} \mathrm{La}$ logica aristotelica, basata sull'Organon (in particolare gli Analitici secondi e il trattato dell'Interpretazione) serviva come impalcatura per l'argomentazione medica e forniva una teoria della causalità; la Filosofia naturale, anch'essa insegnata su Aristotele, toccava temi che oggi chiameremmo scientifici, come il rapporto fra anima e corpo. Conseguita la laurea in Arti, si poteva affrontare la preparazione medica. Le fondamenta aristoteliche e un'educazione ippocratico-galenica - che avevano notevoli affinità - costituivano il nocciolo della formazione. Il medico colto discuteva con gli strumenti della filosofia aristotelica anche le vedute dei commentatori arabi, come Avicenna, Averroè e Haly Abbas. Anche l'insegnamento astrologico era strettamente legato a quello medico, in virtù dell'idea che la posizione dei pianeti esercitasse un'influenza sulla salute degli uomini: formando l'oroscopo del malato, il medico poteva predire il decorso della malattia. ${ }^{28}$

Oltre a istruirci sulla didattica, il manoscritto permette un confronto con gli statuti di altri Collegi medico-artistici e rivela l'irraggiamento di Pavia come polo accademico nell'Italia settentrionale: la

27 Avrebbe peraltro potuto vertere su una o più delle Arti del trivio e del quadrivio.

28 Vd. Ferraresi, A., Il curriculum delle Arti, in Almum Studium Papiense. Storia dell'Università di Pavia, 1.II, 1067-1110; vd. inoltre le sintesi di Kusukawa, S., Medicine in Western Europe in 1500, in Elmer, P., a c. di, The Healing Arts: Health, Disease and Society in Europe (1500-1800), Manchester, Manchester University Press, 2004, 1-26 e Bianchi, L., I contenuti dell'insegnamento: arti liberali e filosofia nei secoli XIII-XVI, in Brizzi, G.P., - Del Negro, P. - Romano, A., a c. di, Storia delle Università in Italia, II, Messina, Sicania, 2007, 117-141: quest'ultimo studioso scrive che «ancor più concisi, gli statuti del Collegio dei dottori in arti e Medicina di Pavia, del 1409, offrivano qualche sommaria indicazione» sulle materie d'insegnamento: il manoscritto rivela ora che l'apparente concisione dello statuto del 1409 dipendeva dall'incompletezza delle edizioni moderne. 
dipendenza degli statuti di Parma e di Torino da quelli pavesi, già ipotizzata prima che si conoscesse la copia completa, viene pienamente confermata. ${ }^{29}$

29 Vd. Rosso, P., in Azzolini, M. et Alii, La Facoltà di Arti e Medicina, in Almum Studium Papiense. Storia dell'Università di Pavia, 1.I, 517; cfr. anche Maierù, A., Ancora sugli atti scolastici nelle università italiane, in Gatto, L. - Supino Martini, P., a c. di, Studi sulle società e le culture del Medio evo per Girolamo Arnaldi, I, Firenze, All'insegna del Giglio, 2002, 308-309, che rileva la sostanziale omogeneità degli esami a Parma, Pisa e Pavia (ma senza disporre dello statuto completo). La rubrica XII (De assignatione punctorum et super quibus libris) compare, con lievissime varianti, negli statuti del Collegio dei dottori artisti e medici di Parma, riformati nel 1415, dopo il passaggio della città alla dominazione di Niccolò III d'Este, avvenuto nel 1412: vd. Gualazzini, U., Corpus Statutorum Almi Studii Parmensis (saec. XV). Con introduzione su "La storia della Università di Parma dalle origini al secolo XV", Milano, Giuffrè, 1978, 54-55 e CLXVIII, nt. 128. Lo stesso vale per il Collegio torinese: Statuta venerandi collegii dominorum artium et medicine doctorum civitatis Taurinensis facta regnante domino duce et principe nostro Ludovico anno Domini 1448 (Torino - Biblioteca Reale, Storia patria 600; cod. membr., sec. XV), c. 11: cfr. Naso, I. - Rosso, P., Insignia doctoralia. Lauree e laureati all'Università di Torino tra Quattro e Cinquecento, Torino, Università degli Studi di Torino, 2008, 32-34: anche questi attenti studiosi - disponendo solo delle edizioni moderne prive della rubrica XII - rilevavano il singolare laconismo dello statuto pavese. Ovviamente, nei contenuti (altro è la forma) gli statuti pavesi a loro volta attingevano a una tradizione ormai consolidata. 


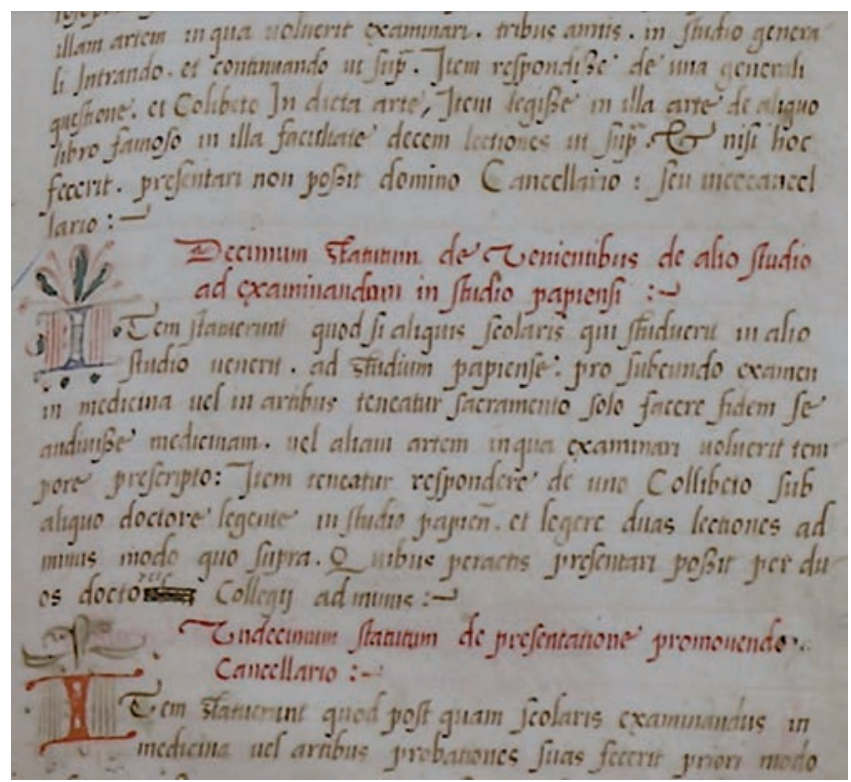

Fig. 1 - Pavia - Museo per la Storia dell'Università, Statuta,

Ordines et Nomina Collegii doctorum Artium et Medicinae Studii Papiensis: dettaglio della c. 3v, con il decimum statutum

(de venientibus de alio Studio ad examinandum in Studio papiensi) non compreso nella copia della Biblioteca Universitaria di Pavia, riprodotta nella Fig. seguente.

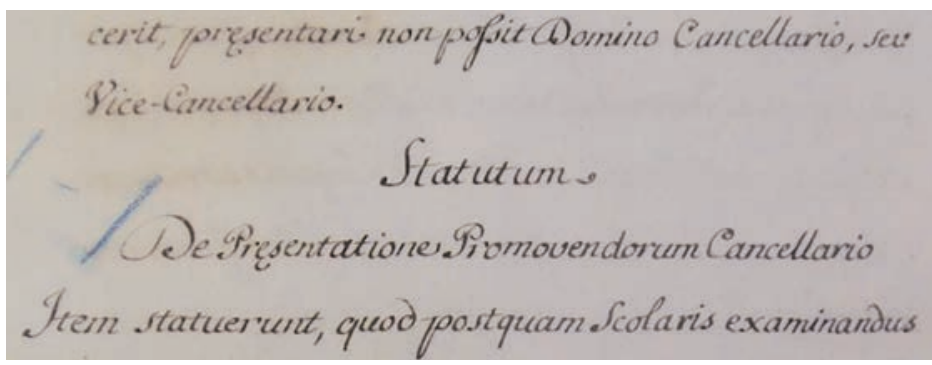

Fig. 2 - Pavia - Biblioteca Universitaria, ms. Ticinesi, 748: dettaglio della p. 15, con le ultime righe del nono statuto e l'incipit dell'undicesimo.

Il segno a matita blu - lasciato da chi compi una successiva ricollazione fra le due copie indica la lacuna. Probabilmente è della stessa mano che ha apposto un segno corrispondente sulla copia più antica,

visibile (nella Fig. 1) in margine a sinistra del capolettera del decimum statutum. 


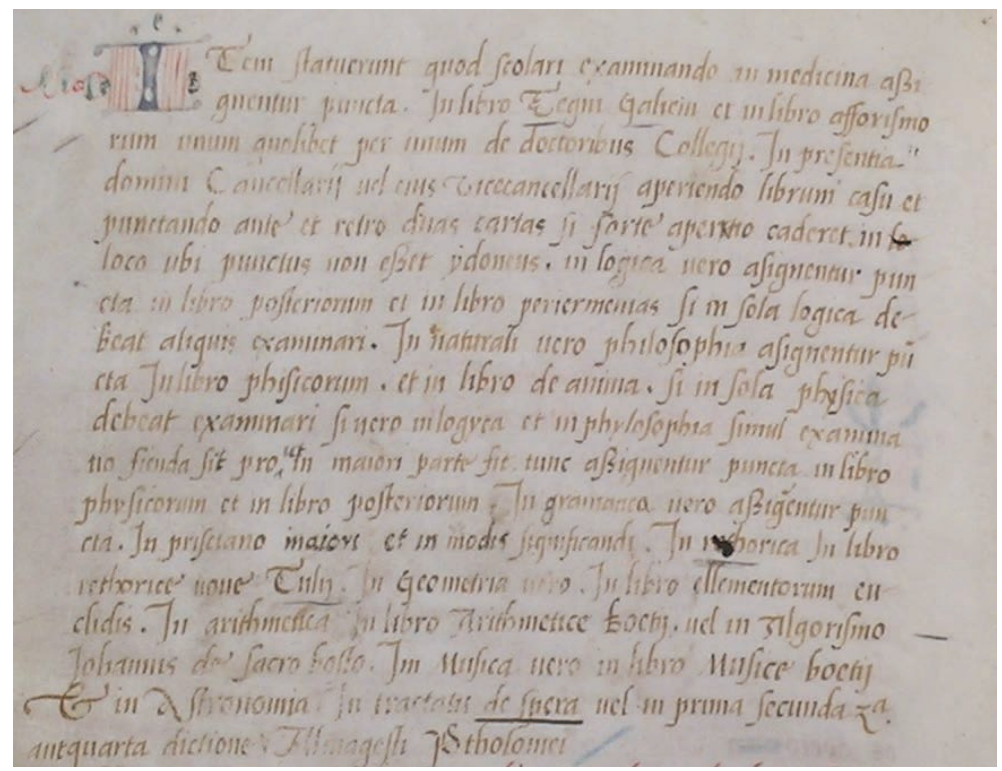

Fig. 3 - Pavia - Museo per la Storia dell'Università, Statuta, Ordines et Nomina Collegii doctorum Artium et Medicinae Studii Papiensis: dettaglio della c. $4 \mathrm{v}$, con il testo del duodecimum statutum

(de assignatione punctorum: la rubrica è nella pagina precedente), relativo ai libri da cui venivano tratti i puncta oggetto dell'esame per il dottorato. 